\title{
A Hybrid Multimodel Neural Network for Nonlinear Systems Identification
}

\author{
I. Baruch*_**, F. Thomas***, R. Garrido*, and E. Gortcheva* \\ *CINVESTAV-IPN, Mexico D.F., MEXICO, \\ E-mail: baruch@ ctrl.cinvestav.mx \\ **IIT-BAS, Sofia, BULGARIA, \\ E-mail: ips@bgearn.acad.bg \\ ***IRI-UPC, Barcelona, SPAIN, \\ E-mail: thomas@iri.upc.es
}

\begin{abstract}
An improved universal parallel recurrent neural network canonical architecture, named Recurrent Trainable Neural Network (RTNN), suited for state-space systems identification, and an improved dynamic back-propagation method of its learning, are proposed. The proposed RTNN is studied with various representative examples and the results of its learning are compared with other results, given in the literature. For a complex non-linear plants identification, a fuzzy-rule-based system and a fuzzyneural multimodel, are used. The fuzzy-neural multimodel is applied for mechanical system with friction identification.
\end{abstract}

\section{Introduction}

The Neural Network (NN) modelling and application to system identification, prediction and control was discussed for many authors [1], [2], [3], [4], [5]. Mainly, two types of NN models are used: Feedforward (FFNN) and Recurrent (RNN). There exists some drawbacks of all described in the literature NN models. As it could be seen in [2], [3], [5], there exists a great variety of $\mathbf{N N}$ models and a universality is missing. All NN models are sequential in nature as implemented for systems identification. The FFNN model uses one or two tap-delays in the input, [1], and RNN models usually are based on the autoregressive model architecture , [2], [5], which is one-layer sequential one. Some of the applied RNN models are not trainable others are not trainable in the feedback part, [4]. Most of them are dedicated to a SISO and not to a MIMO applications, [2]. In more of the cases, the stability of the RNN is not considered, especially during the learning, [4]. In the case of FFNN application for systems identification, the plant model is described by means of four possible nonlinear models, [1]. The linear part of the plant model, especially the system order, has to be known and the FFNN approximates only the nonlinear part of it. The described in the literature learning methods for dynamic NNs are too complicated and difficult to understand, [3]. A new Jordan canonical RNN has been proposed by the authors in its previous paper, [6], and it has been extended to a multimodel case, [7]. They propose a Fuzzy-Neural (FN) approach, extending the Takagi-Sugeno model to resolve more complex identification tasks, [7]. The aim of this paper is to perform a deep topological analysis of the described models and to propose an improved hybrid RTNN model, introducing some weight feedback restrictions to preserve RTNN stability and to confirm its identification abilities. Simultaneously with the improvement of RTNN topology, some advanced researches has been done on the methods of its learning, leading to the design of a new RTNN dynamic Backpropagation (BP) learning method and to its new fuzzy-neural multimodel implementation. The proposed RTNN model for system identification is studied by means of various non-linear discrete-time dynamic objects. Three types of object models are suggested: models, non-linear on their output, state and input. Simulation examples of nonlinear objects and of mechanical object with friction, [8], identified by two RTNN models, coordinated by a rule-based fuzzy system (RBFS), are given.

\section{Description of the RTNN Model and its Learning}

The described in [6] two-layer Jordan canonical RTNN architecture, improved with an additional stability preserving restriction on the feedback weight matrix of the hidden layer, is given for both continuous and discrete time cases in the form: 


$$
\begin{aligned}
& \dot{x}=J x+B u, z=S(x) ; y=S(C z) \\
& \operatorname{Re}\left(J_{i}\right)<0
\end{aligned}
$$

$$
\begin{gathered}
\mathbf{X}(\mathbf{k}+\mathbf{1})=\mathbf{J X}(\mathbf{k})+\mathbf{B U}(\mathbf{k}), \mathbf{Z}(\mathbf{k})=\mathbf{S}[\mathbf{X}(\mathbf{k})] ; \mathbf{Y}(\mathbf{k})=\mathbf{S}[\mathbf{C Z}(\mathbf{k})] \text { (3) } \\
\left|\mathbf{J}_{\mathbf{i}}\right|<\mathbf{1}
\end{gathered}
$$

where the two layer equations of RTNN are separated by semicolon; $\mathbf{y}, \mathbf{x}, \mathbf{u}(\mathbf{Y}, \mathbf{X}, \mathbf{U})$ are output, state and input vectors with dimensions $\mathbf{l}, \mathbf{n}, \mathbf{m}$, respectively; $\mathbf{J}=$ block$\operatorname{diag}\left(\mathbf{J}_{\mathbf{i}}\right)$ is a $(\mathbf{n x n})$ - block-diagonal weight matrix; $\mathbf{J}_{\mathbf{i}}$ are blocks of $\mathbf{J}$ with (1x1) or (2x2) dimensions (equations (2) and (4) are stability conditions, imposed on all blocks $\mathbf{J}_{\mathbf{i}}$ of $\mathbf{J}$, which are in fact conditions on system eigenvalues for both continuous and discrete-time cases); $\mathbf{B}$ and $\mathbf{C}$ are (nxm) and (lxn)- weight matrices; $\mathbf{S}(\mathbf{x})$ is a vector-valued activation function, given as:

$$
\left.S^{\prime}(\operatorname{inp})=\left[s\left(\operatorname{inp}_{1}\right), s_{\left(i n p_{2}\right.}\right), ., s\left(\operatorname{inp}_{j}\right), s\left(\operatorname{inp}_{n}\right)\right]
$$

Here inp is the input variable of the activation function $\mathbf{s}\left(\mathbf{i n p}_{\mathbf{j}}\right)$, which is an element of the vector function $\mathbf{S}$. The activation functions in use are the sigmoid and the saturation function, given by the equations:

$$
\begin{aligned}
& \operatorname{s}(\operatorname{inp})= 1 /[1+\exp (-\operatorname{inp})] ; \operatorname{inp}=\underset{i}{\Sigma}\left(w_{i} x_{i}+w_{i 0}\right) \\
& \operatorname{sat}(\operatorname{inp})=\left\{\begin{array}{l}
+1, \text { inp } \geq+1 \\
\text { inp, } 0 \leq \operatorname{inp}<+1 \\
0, \quad \text { inp }<0
\end{array}\right.
\end{aligned}
$$

where $\mathbf{w}_{\mathbf{i}}, \mathbf{w}_{\mathbf{i o}}$ are trainable weights of the RTNN; $\mathbf{S}^{\prime}$ signifies a vector transpose of $\mathbf{S}$. The saturation function is used as approximation of the sigmoid function to improve the RTNN architecture, facilitating its realisation.

The three layer versions of the RTNN continuous and discrete-time architecture are as follows:

$$
\begin{gathered}
\dot{\mathbf{x}}=\mathbf{J x}+\mathbf{q}, \mathbf{z}=\mathbf{S}(\mathbf{x}) ; \mathbf{q}=\mathbf{S}(\mathbf{B u}) ; \mathbf{y}=\mathbf{S}(\mathbf{C z}) \\
\mathbf{X}(\mathbf{k}+\mathbf{1})=\mathbf{J X}(\mathbf{k})+\mathbf{Q}, \mathbf{Z}(\mathbf{k})=\mathbf{S}[\mathbf{X}(\mathbf{k})] ; \mathbf{Q}=\mathbf{S}[\mathbf{B U}(\mathbf{k})] ; \\
\mathbf{Y}(\mathbf{k})=\mathbf{S}[\mathbf{Z}(\mathbf{k})]
\end{gathered}
$$

where $\mathbf{q},(\mathbf{Q})$ is a $\mathbf{n}$ - input vector of the hidden layer.

The given above RTNN model (in two and three layered versions) could be linearised and its dynamic behaviour could be studied by the first stability law of Liapunov, where the stability conditions, (2), (4), for continuous and discrete time state-space models, could be used. Than it is easy to analyse the $\mathbf{N N}$ model controllability, observability and identifiability. From the block structure of $\mathbf{B}$ and $\mathbf{C}$, corresponding to the block structure of $\mathbf{J}$, we can conclude if the RTNN could be learned or not, [6]. The main advantages of the proposed three layer Jordan Canonical Form (JCF) RTNN architecture, defined for both continuous and discrete-time cases are that the described JCF RTNN model is an universal hybrid neural model which contains one or two FF layers and one recurrent hidden layer with completely decomposed dynamics, as the matrix $\mathbf{J}$ is block-diagonal. So, it has a minimum number of parameters and it is completely parallel, as the Jordan canonical form is parallel with respect to the regressive model, which is a sequential one. The RTNN architecture is described in state-space form (SISO or MIMO) and could serve as one-step ahead state predictor/estimator. The RTNN model is non-linear in large and linear in small, so the matrices $\mathbf{J}, \mathbf{B}, \mathbf{C}$, obtained as a result of learning, could be used for analytical design of linear state/output feedback/feedforward control laws. Finally, the RTNN could solve the optimal control problem itself by means of a NN mapping , [1]. The obtained RTNN model is a robust model, as the learning method applied for weights adjustment is a dynamic BP method, which is based on the network sensitivity model, [1]. It also permits to perform node pruning and weight fixing during the learning.

The most common used BP updating rule, applied for the two-layer RTNN canonical model, [6], is the following:

$$
\mathbf{W}_{\mathrm{ij}}(\mathbf{k}+\mathbf{1})=\mathbf{W}_{\mathrm{ij}}(\mathbf{k})+\eta \Delta \mathbf{W}_{\mathrm{ijj}}(\mathbf{k})
$$

where: $\mathbf{W}_{\mathbf{i j}}$ is a general weight, denoting the $\mathbf{i j}$-th weight element of each weight matrix $(\mathbf{C}, \mathbf{J}, \mathbf{B})$ in the RTNN model to be updated; $\Delta \mathbf{W}_{\mathrm{ij}},\left(\Delta \mathbf{C}_{\mathrm{ij}}, \Delta \mathbf{J}_{\mathrm{ij}}, \Delta \mathbf{B}_{\mathrm{ij}}\right)$, is the weight correction of $\mathbf{W}_{\mathrm{ij}} ; \eta$ is the learning rate parameter. If there are some oscillations in the error during the learning, a momentum term can be added in the weight updating rule (10). One of the following two forms can be used:

$$
\begin{gathered}
\mathbf{W}_{\mathrm{ij}}(\mathbf{k}+\mathbf{1})=\mathbf{W}_{\mathrm{ij}}(\mathbf{k})+\eta \Delta \mathbf{W}_{\mathrm{ij}}(\mathbf{k})+\alpha \Delta \mathbf{W}_{\mathrm{ij}}(\mathbf{k}-\mathbf{1}) \\
\mathbf{W}_{\mathrm{ij}}(\mathbf{k}+\mathbf{1})=\mathbf{W}_{\mathrm{ij}}(\mathbf{k})+\eta(\mathbf{1}-\alpha) \Delta \mathbf{W}_{\mathrm{ij}}(\mathbf{k})+\alpha \Delta \mathbf{W}_{\mathrm{ij}}(\mathbf{k}-\mathbf{1})
\end{gathered}
$$

where $\alpha$ is a momentum term learning rate parameter. The equation (12) establish some inverse dependence between the learning parameters of the first and second updating terms. A lot of experiments of learning with different rates of learning $\eta$ and $\alpha$ in equation (11), has been done. These experiments show that the optimal combination of these learning parameters is obtained when the following inequality condition yields:

$$
r_{\max }<\operatorname{sqrt}\left(\eta^{2}+\alpha^{2}\right)<1 ; r_{\max }=\max \left|\lambda_{i}\right|
$$

where $\max \left|\lambda_{\mathbf{i}}\right|$ is the maximum eigenvalue of the identified plant model. The experimentally obtained rule (13) shows that the circle with radius $\mathbf{r}$, which depends on the values 
of $\eta$ and $\alpha\left(\mathbf{r}=\operatorname{sqrt}\left(\eta^{2}+\alpha^{2}\right)\right)$, must contain all eigenvalues of the matrix $\mathbf{J}$ of the discrete-time RTNN model (2). The weight corrections of the updated matrices in the discretetime RTNN model, described by eqn. (2), are performed using the following update equations:

- For the output layer:

$$
\Delta C_{i j}(k)=\left[T_{j}(k)-Y_{j}(k)\right] Y_{j}(k)\left[1-Y_{j}(k)\right] Z_{i}(k)
$$

where: $\Delta \mathbf{C}_{\mathbf{i j}}$ is the weight correction of the $\mathbf{i j}$-th elements of the (Ixn) learned matrix $\mathbf{C}$; $\mathbf{T}_{\mathbf{j}}$ is a $\mathbf{j}$-th element of the target vector; $\mathbf{Y}_{\mathbf{j}}$ is a $\mathbf{j}$-th element of the output vector; $\mathbf{Z}_{\mathbf{i}}$ is an $\mathbf{i}$-th element of the output vector of the hidden layer.

- For the hidden layer:

$$
\begin{aligned}
& \Delta \mathbf{B}_{\mathrm{ij}}(\mathbf{k})=\mathbf{R} \mathbf{U}_{\mathbf{i}}(\mathbf{k}) \\
& \Delta \mathbf{J}_{\mathrm{ij}}(\mathbf{k})=\mathbf{R} \mathbf{X}_{\mathbf{i}}(\mathbf{k - 1}) \\
& \mathbf{R}=\mathbf{C}_{\mathbf{i}}(\mathbf{k})[\mathbf{T}(\mathbf{k})-\mathbf{Y}(\mathbf{k})] \mathbf{Z}_{\mathbf{j}}(\mathbf{k})\left[1-\mathbf{Z}_{\mathbf{j}}(\mathbf{k})\right]
\end{aligned}
$$

where: $\Delta \mathbf{B}_{\mathbf{i j}}$ is the weight correction of the $\mathbf{i j}$-th elements of the $(\mathbf{m} \times \mathbf{n})$ learned matrix $\mathbf{B} ; \mathbf{C}_{\mathbf{i}}$ is a row vector of dimension $(\mathbf{1} \mathbf{x l})$, taken from the transposed matrix $\mathbf{C}^{\prime}$; [T-Y] is a $(\mathbf{I X 1})$ output error vector, through which the error is back-propagated to the hidden layer; $\mathbf{U}_{\mathbf{i}}$ is an $\mathbf{i}$-th element of the input vector $\mathbf{U} ; \mathbf{X}_{\mathbf{i}}$ is an $\mathbf{i}$-th element of the vector $\mathbf{X} ; \Delta \mathbf{J}_{\mathbf{i j}}$ is the weight correction of the $\mathbf{i j}$-th elements of the (nxn) block-diagonal matrix $\mathbf{J}$ under learning; $\mathbf{R}$ is an auxiliary variable. The same equation for RTNN learning may be applied for the continuous-time case, given by equation (1). Another improvement of the RTNN learning algorithm, successfully applied for the BP learning of discrete-time RTNNs consider unimportant units pruning and non-useful connections removing, which lead to exclusion of weights or nodes from the process of learning. The improved learning algorithm for RTNN was tested with several linear and non-linear dynamic objects. The topology improvements are also carefully studied. The applicability of the improved RTNN model for system identification and prediction is illustrated by appropriate example of non-linear dynamic system.

\section{A Fuzzy System Rule Based Coordination Model}

Let us assume that the unknown system $\mathbf{y}=\mathbf{f}(\mathbf{x})$ generates the data $\mathbf{y}(\mathbf{k})$ and $\mathbf{x}(\mathbf{k})$ measured at $\mathbf{k}, \mathbf{k}-\mathbf{1}, . .$. , then the aim is to use this data to construct a deterministic function $\mathbf{y}=\mathbf{F}(\mathbf{x})$ that can serve as a reasonable approximation of $\mathbf{y}=\mathbf{f}(\mathbf{x})$ in which the function $\mathbf{f}(\mathbf{x})$ is unknown.

The variables $\mathbf{x}=[\mathbf{x} 1, \ldots, \mathbf{x p}]^{\prime} \in \mathfrak{\aleph} \subset \mathfrak{R}^{\mathrm{p}}$ and $\mathbf{y} \in \mathrm{Y} \subset \mathfrak{R}$ are called regressor and regressand, respectively. In Fuzzy
System (FS) modelling, the function $\mathbf{F}(\mathbf{x})$ is represented as a collection of if-than fuzzy rules of the type:

\section{IF antecedent proposition then consequent proposition}

The Takagi-Sugeno model, cited in [7], is a mixture between a linguistic and mathematical regression models. The rule antecedents describes the fuzzy regions in the input space. The rule consequent is a crisp mathematical function of the inputs. This mathematical function could be a dynamic function too, given by a state-space mathematical model of the form:

$$
\begin{aligned}
& R_{i} \text { : If } \mathrm{x}(\mathrm{k}) \text { is } \mathrm{J}_{\mathrm{i}} \text { and } \mathrm{u}(\mathrm{k}) \text { is } \mathrm{B}_{\mathrm{i}} \\
& \text { then }\left\{\begin{array}{l}
\mathrm{x}_{\mathrm{i}}(\mathrm{k}+1)=\mathrm{J}_{\mathrm{i}} \mathrm{x}(\mathrm{k})+\mathrm{B}_{\mathrm{i}} \mathrm{u}(\mathrm{k}) \\
\mathrm{yi}(\mathrm{k}) \quad=\mathrm{C}_{\mathrm{i}} \mathrm{x}(\mathrm{k})
\end{array}\right.
\end{aligned}
$$

Our proposal is that this function be a RTNN model, given by equation (2). So, the FS model obtains the form:

$$
R_{i}: \text { If } \mathrm{x} \text { is } \mathrm{J}_{\mathrm{i}} \text { and } \mathrm{u} \text { is } \mathrm{B}_{\mathrm{i}} \text { then } \mathrm{y}_{\mathrm{i}}=\mathrm{N}_{\mathrm{i}}(\mathrm{x}), \mathrm{i}=1,2, . .
$$

where the consequent function $\mathbf{y}_{\mathbf{i}}=\mathbf{N}_{\mathbf{i}}(\mathbf{x})$ represents the RTNN model given by the equation (2). To incorporate the RTNN model (2) into the FS model (19), it have to use the RTNN model (2) in the consequent proposition part of (19) and the restrictions, expressed by the saturation of (7) to construct the antecedent proposition part of (19). The biases, obtained in the process of $\mathbf{B P}$ learning of the RTNN model could be used to form membership functions, as they are natural centres of gravity, [7], for each variable. The number of rules could be optimised using the mean-square error (MSE) of RTNNs learning, which is a natural measure of precision of the approximation of the non-linear object sub-model (MSE is up to 3\%). The structure of the entire identification system contains a fuzzyfier a Fuzzy Rule-Based System (FRBS), and a set of RTNN models. The system does not need a defuzzyfier, because RTNN models are crisp limited linear state-space models. A possible adaptive control system, could contain also a set of controllers incorporated in a FRBS, designed on the base of the obtained set of RTNNs.

\section{Simulation Results}

\subsection{Example 1}

The first example, taken from [3], is a discrete-time model of nonlinear object with input and output smooth nonlinearities, identified by one RTNN model. The learning signal, is chosen as sequence of pulses with random amplitude and width. The neural model quality is 
verified comparing reactions of the plant and the RTNN to an unknown generalisation signal of one-epoch length. This signal, taken from [3], has the form:

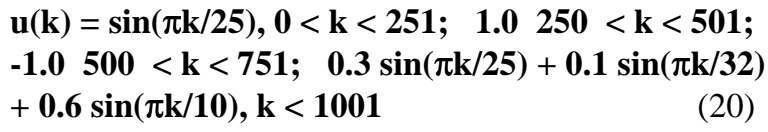

The SISO discrete-time nonlinear plant model is described by the following equations, [3]:

$$
\begin{aligned}
& \mathrm{x}_{1}(\mathrm{k}+1)=\mathrm{x}_{2}(\mathrm{k}) \\
& \mathrm{x}_{2}(\mathrm{k}+1)=-0.15 \mathrm{x}_{1}(\mathrm{k})+0.8 \mathrm{x}_{2}(\mathrm{k})+\mathrm{h}(\mathrm{k}) \\
& \mathrm{h}(\mathrm{k})=3 \mathrm{u}_{1}{ }^{3}(\mathrm{k}) /\left[1+4 \mathrm{u}_{1}{ }^{2}(\mathrm{k})\right] \\
& \mathrm{u}_{1}(\mathrm{k})=1.9 \mathrm{u}(\mathrm{k}) \\
& \mathrm{y}(\mathrm{k}+1)=\left\{\left[0.3 \mathrm{x}_{1}(\mathrm{k}+1)+\mathrm{x}_{2}(\mathrm{k}+1)\right] / 3.5\right\}^{3}
\end{aligned}
$$

Simulation results, obtained for RTNN identification model, using sigmoid activation function and BP learning algorithm with momentum term, are given in the Fig.1
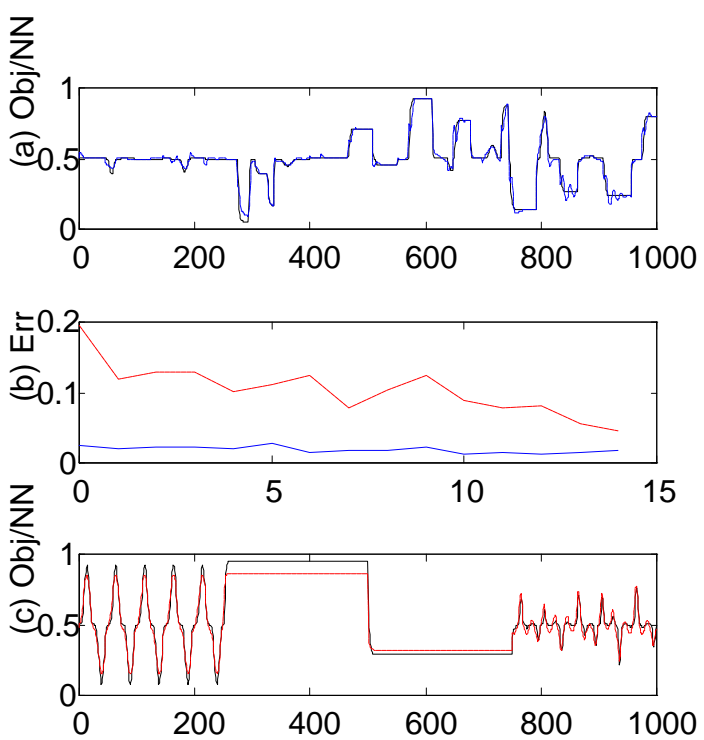

Fig.1. RTNN architecture $(1,7,1), \eta=0.5, \alpha=0.75$, sigmoid activation function, MSEG $=4 \%$, MSEL $=2 \%$, $\mathbf{N}=14$ epochs.

(a) Output of RTNN (dashed line) and Object (solid line) during last epoch of training (1000 iterations).

(b) RTNN MSEG error of generalisation (dashed line) and training (solid line) for all epochs of learning

(c) Output of RTNN (dashed line) and Object (solid line) during last epoch of generalisation.

The RTNN architecture $(\mathbf{1}, \mathbf{7}, \mathbf{1})$ applied, has one input, seven neurones in the hidden layer and one output. The time of learning is $\mathbf{N}=\mathbf{1 4}$ epochs (one epoch contain $\mathbf{1 0 0 0}$ iterations) and the final Mean Square Errors (MSE) of learning and generalisation obtained are MSEL $=\mathbf{2 \%}$, MSEG $=\mathbf{4 \%}$, respectively. The obtained results in [3] for the same examples are: time of learning between $\mathbf{6 2} 000$ and 77000 iterations which is about 5 times longer with respect to RTNN. The means square error, obtained in [3] for architecture (3:0) is $\mathbf{3 . 4 5 \%}$ and for architecture (6:1) is 6.41\%. Note that in [3], an FFNN architecture with memory neurones is used, which duplicates the number of neurones required. So the first architecture (the better one) uses 1 network neurone and $\mathbf{1}$ memory neurone in the input layer, $\mathbf{3}$ network neurones plus $\mathbf{3}$ memory neurones in the hidden layer, and $\mathbf{1}$ network neurone and $\mathbf{0}$ memory neurones in the output layer. The total number of neurones required for this NN is $\mathbf{9}$, and for RTNN it is 8 .

\subsection{Example 2}

The second example is a discrete-time model of nonlinear 1- DOF mechanical object with friction, taken form [8]. The discrete-time model of the 1-DOF mass mechanical system with friction, is given in the form:

$$
\begin{aligned}
& x_{1}(k+1)=x_{2}(k) \\
& x_{2}(k+1)=-0.025 x_{1}(k)-0.3 x_{2}(k)+0.8 u(k)-0.1 f r(v, k)
\end{aligned}
$$

where: $\mathbf{x}_{\mathbf{1}}(\mathbf{k}), \mathbf{x}_{\mathbf{2}}(\mathbf{k})$ are system states; $\mathbf{k}$ is a discrete time variable and the friction force $\mathbf{f r}(\mathbf{v}, \mathbf{k})$, [8], is assumed to be modelled as follows:

$$
\begin{aligned}
& \mathbf{f r}(\mathbf{v}, \mathbf{k})=\mathbf{F}_{\text {slip }}(\mathbf{v}) \mathbf{a}(\mathbf{v})+\mathbf{F}_{\text {stick }}(\mathbf{u})[\mathbf{1}-\mathbf{a}(\mathbf{v})] \\
& \mathbf{a}(\mathbf{v})=\left\{\begin{array}{c}
+1,|\mathbf{v}(\mathbf{k})|>\alpha \\
; \alpha>0 \\
0,|\mathbf{v}(\mathbf{k})| \leq \alpha
\end{array}\right.
\end{aligned}
$$

The sticking friction provides the value of the friction forces at zero velocity $\mathbf{v}$. The term is used to describe whether the mass will stick or break free from the static friction forces. The positive and negative limits on the static friction forces are given by $\mathbf{F}_{\mathrm{s}}^{+}$and $\mathbf{F}_{\mathrm{s}}^{-}$, respectively. Generally, they are not equal in magnitude, and the model should consider these asymmetry. It is modelled as follows:

$$
\mathbf{F}_{\text {stick }}(\mathbf{u})= \begin{cases}\mathbf{F}_{\mathrm{s}}^{+}, & \mathbf{u}(\mathbf{k}) \geq \mathbf{F}_{\mathrm{s}}^{+} \\ \mathbf{u}(\mathbf{k}), & \mathbf{F}_{\mathrm{s}}^{-}<\mathbf{u}(\mathbf{k})<\mathbf{F}_{\mathrm{s}}^{+} \\ \mathbf{0 ,} & \mathbf{u}(\mathbf{k}) \leq \mathbf{F}_{\mathrm{s}}^{-}\end{cases}
$$

The mass cannot move until the applied force is greater in magnitude than the respective static friction force. The slipping function $\mathbf{F}_{\text {slip }}(\mathbf{v})$ provides values of the friction at non-zero velocity and is represented by:

$$
F_{\text {slip }}(\mathbf{v})=F_{d}^{+}(\mathbf{v}) \mathbf{b}(\mathbf{v})+\mathbf{F}_{\mathrm{d}}^{-}(\mathbf{v}) \mathbf{b}(-\mathbf{v})
$$




$$
\begin{aligned}
& \mathbf{b}(\mathbf{v})=\left\{\begin{array}{l}
1, \mathbf{v}(\mathbf{k})>0 \\
0, \mathbf{v}(\mathbf{k}) \leq 0
\end{array}\right. \\
& \mathbf{F}_{\mathrm{d}}^{+}(\mathbf{v})=\mathbf{F}_{\mathrm{s}}^{+}-\Delta \mathbf{F}^{+}\left[1-\exp \left(-\mathrm{v} / \mathbf{v}_{\mathrm{cr}}^{+}\right)\right]+\beta^{+} \mathbf{v} \\
& \mathbf{F}_{\mathrm{d}}^{-}(\mathbf{v})=\mathbf{F}_{\mathrm{s}}^{-}-\Delta \mathbf{F}^{-}\left[1-\exp \left(-\mathbf{v} / \mathbf{v}_{\mathrm{cr}}^{-}\right)\right]+\beta^{-} \mathbf{v}
\end{aligned}
$$

where $\Delta \mathbf{F}^{+}$and $\Delta \mathbf{F}^{-}$are the respective drops from the static to the kinetic force level; $\mathbf{v}_{\mathbf{c r}}{ }^{+}$and $\mathbf{v}_{\mathbf{c r}}{ }^{-}$are the critical Stribeck velocities, and $\beta^{+}$and $\beta^{-}$are the viscous friction coefficients. The friction force is modelled as a summation of the Coulomb friction, viscous friction, and the Stribeck effect. The Stribeck effect is that the friction force is decreasing with increasing fluid lubrication. Some models consider dynamic lag effect of the friction force with respect to the velocity, which effect could be neglected. For sake of simplicity, some slip friction parameters for both velocity directions could be considered as equal (e.g. $\mathbf{v}_{\mathbf{c r}}{ }^{+}$ $=\mathbf{v}_{\mathbf{c r}}{ }^{-}=\mathbf{v}_{\mathbf{c r}} ; \beta^{+}=\beta^{-}=\beta$ ). The 1-DOF mechanical system, [8], is consider to have the following friction parameters: $\alpha$ $=0.001 \mathrm{~m} / \mathrm{s} ; \mathrm{F}_{\mathrm{s}}^{+}=4.2 \mathrm{~N} ; \mathrm{F}_{\mathrm{s}}{ }^{-}=\mathbf{- 4 . 0} \mathrm{N} ; \Delta \mathrm{F}^{+}=1.8 \mathrm{~N} ; \Delta \mathrm{F}^{-}$ $=-1.7 \mathrm{~N} ; \mathrm{v}_{\mathrm{cr}}=0.1 \mathrm{~m} / \mathrm{s} ; \boldsymbol{\beta}=\mathbf{0 . 5} \mathrm{Ns} / \mathrm{m}$. Simulation results, obtained for this plant, identified by two RTNN models, coordinated by a FRBS, are given in Fig.2 and Fig.3. The epoch time is $\mathbf{1 0 0}$ iterations and the learning signal is a sum of sinusoids with different frequencies, as follows:
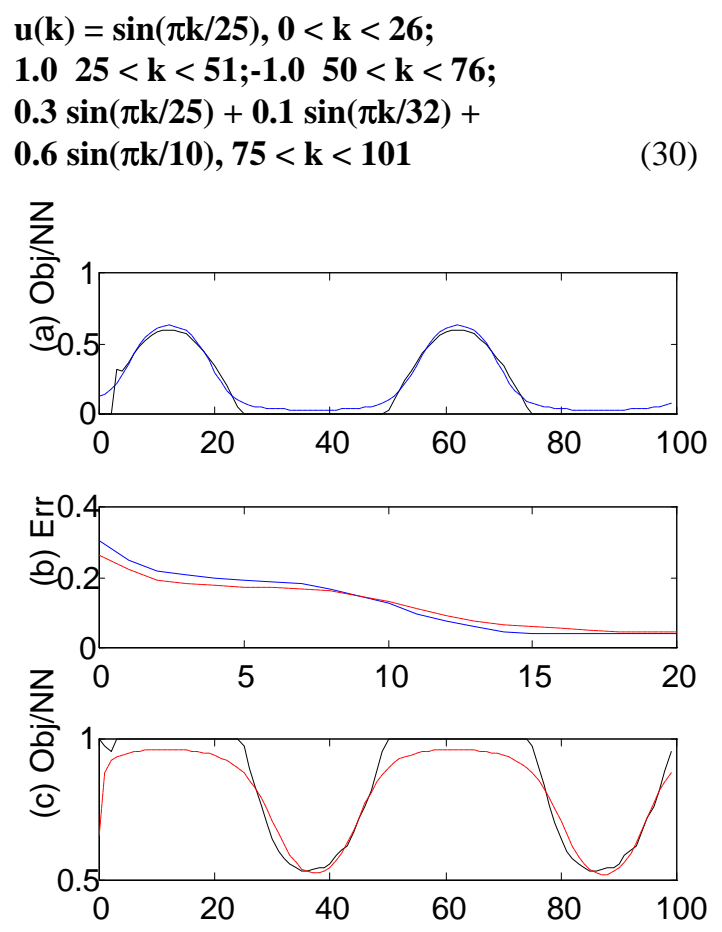

Fig.2. Two RTNN model approach, $\eta=0.2, \alpha=0$.9, sigmoid activation function (a) Output of the first (positive) RTNN (dashed line) and Object (solid line) during last epoch of learning (100 iterations).

(b) RTNN MSE of learning the second RTNN (dashed line) and learning the first RTNN (solid line) for all epochs. Time of learning - 20 epochs. MSE for last epoch of learning - 3\%.

(c) Output of the second (negative) RTNN (dashed line) and Object (solid line) during last epoch of learning (100e iterations.).
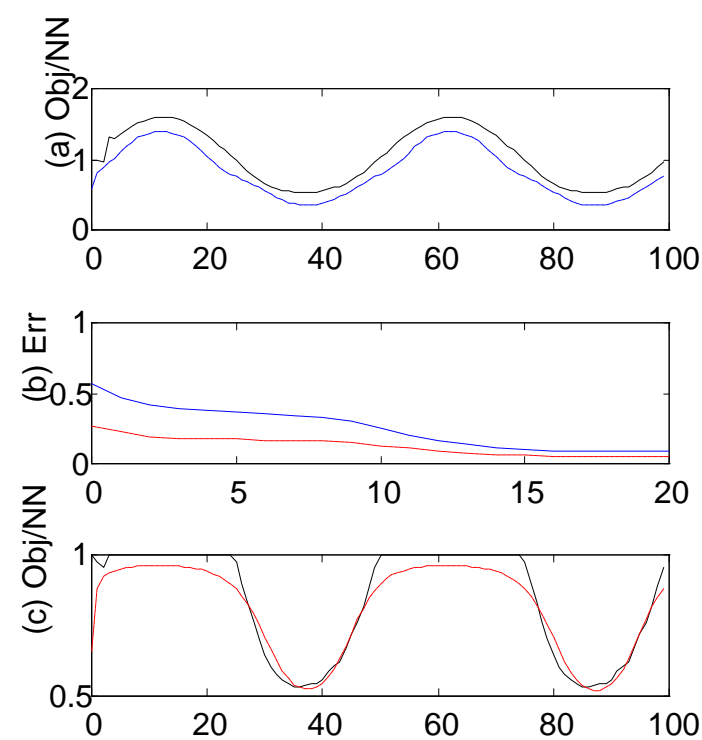

Fig.3. Two RTNN model approach, $\eta=0.2, \alpha=0.9$, sigmoid activation function

(a) Output of the combined fuzzy-neural model (dashed line) and Object (solid line) during last epoch of learning (100 iterations).

(b) RTNN MS error of learning the second RTNN (dashed line) and learning the combined fuzzy-neural model (solid line) for all epochs. Time of learning - 20 epochs. MSE for last epoch of learning - 3\%.

(c) Output of the second (negative) RTNN (dashed line) and Object (solid line) during last epoch of training (100 iterations.).

The first and the third graphics of Fig.2. show the training results of both RTNN models during the last epoch of training (for positive and negative output signals, separately). It compare the positive / negative output of the system with the output of the corresponding RTNN model. The second graphics shows the Mean-Square-Error (MSE) of training for both RTNN models during all the period of training, given in epochs. The results show an excellent 20 epochs convergence with $\mathbf{3 \%}$ MSE for both models. The first graphics of Fig.3. show the training results of the combined fuzzy-neural multi-model during the last epoch 
of training. It compare the output of the system with the output of the combined neural multi-model. For sake of comparison, the third graphics shows again the results given in Fig.2.c. for the second (negative output) RTNN. The second graphics of Fig.3. shows the MSE of the combined and the second models during all the period of learning. The results show an excellent 20 epochs convergence with the same 3\% MSE.

\section{Conclusions}

An improved universal parallel recurrent neural network canonical architecture, named Recurrent Trainable Neural Network, suited for state-space systems identification, and an improved dynamic back-propagation method of its learning, are derived. The obtained hybrid RTNN model is studied with a representative example and the results of its learning and generalisation are compared with other results, given in the literature. The comparison shows the better performance of the RTNN. For a complex non-linear plants identification, a fuzzy-rule-based system and a fuzzyneural multimodel, are used. The fuzzy-neural multimodel is applied for 1-DOF mass mechanical system with friction identification. The result obtained for two RTNN model identification of this complex object also shows a good performance of both RTNN during the learning.

\section{Acknowledgements}

This work has been partly supported by the CINVESTAVIPN, Mexico D.F., MEXICO, IRI-UPC, Barcelona, SPAIN and NSF, MEST, grant 611/1996, Sofia, BULGARIA.

\section{References}

1. K.S.Narendra, and K. Parthasarathy, Identification and Control of Dynamic Systems using Neural Networks, IEEE Transactions on NNs, 1(1), 1990, 4-27.

2. J.T.Connor, R. Martin, and L. Atlas, Recurrent NN and Robust Time Series Prediction. IEEE Transactions on NNs, 5(2), 1994, 240-253.

3. P.S.Sastry, G. Santharam, and K.P. Unnikrishnan, Memory Networks for Identification and Control of Dynamical Systems. IEEE Transactions on NNs, 5(2), 1994, 306-320.

4. D.T.Pham,. and S. Yildirim, Robot Control using Jordan Neural Networks, Proc. of the Internat. Conf. on Recent Advances in Mechatronics, Istanbul, Turkey, Aug. 14-16, 1995, (O. Kaynak, M. Ozkan, N. Bekiroglu, I. Tunay, Eds., Bogaziçi University Printhouse, Istanbul, Turkey, 1995), Vol. II, 888-893.

5. A.C.Tsoi, and A.D. Back, Locally Recurrent Globally Feedforward Networks: A Critical Review of Architectures. IEEE Transactions on NNs, 5(2), 1994, 229-239.

6. I.Baruch, I. Stoyanov, and E. Gortcheva, Neural Network Models of Dynamic Processes: Stability and Learning, Proc. of the 3-rd Int. Symp. MMAR'96, Sept. 10-13, 1996, Miedzyzdroje, Poland, (S. Banka, S. Domek, Z. Emirsajlov, Eds., TU of Szczecin, Poland, 1996), Vol. 3, pp. 1169-1174.

7. I.Baruch, and E.Gortcheva, Fuzzy-Neural Model for Nonlinear Systems Identification, Proc. of the 5-th IFAC Workshop AARTC'98, April 15-17, 1998, Cancun, Mexico, (D.G.Nocetti, J.S. Gonzalez, P.A. Contla, P.J.Fleming, Eds., AMCA, Mexican Society of Automatic Control,1998), 283288.

8. Seon - Woo Lee, and Jong-Hwan Kim, Robust Adaptive Stick-Slip Friction Compensation, IEEE Transactions on IE, 42(5), 1995, 474-479. 